\title{
El entorno social-familiar en la vulneración de los derechos de los niños con discapacidad
}

\section{The social-family environment in the violation of the rights of children with disabilities}

Rously Eedyah Atencio-González

uq.rouslyatencio@uniandes.edu.ec

Universidad Regional Autónoma de los Andes, Quevedo

Ecuador

https://orcid.org/0000-0001-6845-1631

Angélica Narcisa Fernández-Aguilar

dq.angelicanfa36@uniandes.edu.ec

Universidad Regional Autónoma de los Andes, Quevedo

Ecuador

https://orcid.org/0000-0003-3597-7332

Daniel Eduardo Fernández-Aguilar

dq.danielef66@uniandes.edu.ec

Universidad Regional Autónoma de los Andes, Quevedo

Ecuador

https://orcid.org/0000-0003-2111-4958

Rudy Gabriela Yong-Zambrano

dq.rudygyz21@uniandes.edu.ec

Universidad Regional Autónoma de los Andes, Quevedo

Ecuador

https://orcid.org/0000-0002-7206-9379

Recepción: 15 de septiembre 2021

Revisado: 25 octubre 2021

Aprobación: 15 de noviembre 2021

Publicación: 01 de diciembre 2021 


\section{Estimado Editor (a):}

El concepto de discapacidad ha venido evolucionando en los últimos años, pues el mismo ya no se lo determina como un enfoque biomédico, hoy en día es considerado una garantía constitucional, el mismo que se encuentra incluido en la norma constitutiva y organizativa de un Estado preceptuado en la Constitución. Los derechos humanos son imprescindibles para la vida del hombre en la sociedad es decir que estos derechos están conectados a la persona dentro del Estado y sociedad.

En este sentido, la Carta Suprema del Estado eleva a derechos fundamentales, en el año 2008 las personas con discapacidad vivieron un cambio radical con la vigencia de la actual Carta Magna en donde se incluyeron la garantía de los derechos de las personas con discapacidad, creándose así normas públicas y políticas en las que se debía garantizar la participación de las personas con discapacidad para el pleno goce de sus derechos siendo estos: salud, educación, empleo, capacitación, empleo y exenciones tributarias. Del tal modo se crearon instituciones como el Ministerio de Inclusión Económica y Social (MIES), en donde su finalidad era incluir la inclusión económica y social para todas las personas con discapacidad y su familia, cuya gestión era el cumplir con los principios de igualdad, integralidad, corresponsabilidad, inclusión y equidad en la sociedad.

Por lo tanto, los derechos de las personas y familias con discapacidad se encuentran contemplados en normativas nacionales siendo esta en Ecuador la (Constitución de la República del Ecuador, 2008), la (Ley Orgánica de Discapacidades,2012), y de igual manera el reglamento de la misma, en normativas internacionales encontramos la (Convención sobre los Derechos de las Personas con Discapacidad 2006), la (Convención Interamericana para la Eliminación de Todas las formas de Discriminación contra las Personas con Discapacidad 1999), encontrándose así con normativas amplias y suficientes para el pleno goce y uso de sus derechos.

A nivel mundial los niños, niñas y adolescentes con discapacidad, son uno de los grupos más marginados y excluidos dentro de la sociedad, donde sus derechos son vulnerados de manera generalizada. En comparación con sus pares sin discapacidad, tienen más probabilidades de experimentar las consecuencias de la inequidad social, 
cultural y económica a la que a diario se enfrentan a actitudes negativas, estereotipos, estigma, violencia, abuso y aislamiento.

De igual forma, aunado a esto, la falta de políticas públicas en donde existen leyes adecuadas que conlleven a desarrollar oportunidades educativas y económicas en su ámbito. Desafortunadamente los niños, niñas y adolescentes con discapacidad son sujetos al abandono, la negligencia o a su reclusión en instituciones. A esta problemática se le suma la pobreza y la discapacidad, las cuales están estrechamente relacionadas, la primera es un factor determinante de la segunda y a su vez, la discapacidad sumerge a las personas en la pobreza.

En este orden de ideas, los derechos de los niños, niñas y adolescentes con discapacidad se han convertido en un tema fundamental, tal como lo evidencia la (Ley Orgánica de discapacidades, 2012) sin embargo, en la realidad de Ecuador se observa una total despreocupación, por la falta de programas en donde se faciliten la inclusión y concienciación social, para garantizar el pleno goce de sus derechos. El Estado, como tema principal y fundamental debe trabajar por el ejercicio y goce de los derechos de las personas con discapacidad por pertenecer a un grupo de atención prioritaria.

Así mismo en la Convención sobre los Derechos de las Persona con Discapacidad, (2018) ha determinado que las personas con discapacidad siguen encontrando barreras para participar en igualdad de condiciones con los demás ciudadanos, y se les siguen vulnerando sus derechos humanos y constitucionales en todas las partes del mundo, a su vez reconoce que las mujeres y las niñas con discapacidad corren un riesgo mayor dentro y fuera de su hogar, en donde existen violencia, lesiones, abusos, abandono y malos tratos o explotaciones.

Por otro lado, la República del Ecuador como Estado constitucional de derechos, a través de su marco constitucional y diversas normas ha establecido el respeto, protección y la garantía de derechos humanos, el mismo que consagra la atención prioritaria para las personas con discapacidad y en virtud de aquello es fundamental entender la aplicación del principio de igualdad y no discriminación en cada una de sus políticas. En este sentido, consagra los derechos para este grupo, dentro del 
capítulo tercero, el mismo que define a las personas y grupos de atención prioritaria. En su artículo 44 de la mencionada Constitución ha manifestado como obligación del Estado brindar protección y promover el desarrollo integral de niños, niñas y adolescentes. Por su parte, Ayala(2020) define al entorno social, como un espacio constituido por todos los elementos creados por el ser humano, los mismo que rodean a los individuos e interactúan con ellos dentro de la sociedad, ámbito o ambiente social en el que nace y crece el individuo. El entorno social es una serie de elementos creados por el ser humano, en el mismo se indica el ambiente en el que se desenvuelve, siendo este social y cultural ya que son parte de su día a día. Por otro lado, la familia es el nivel micro de la sociedad, dentro de ese grupo de personas que guían el funcionamiento de la familia y crean comportamientos dentro del grupo de la sociedad, a esto se lo entiende como entorno familiar. (Anabella Coria, 2012)

En este sentido, según Valencia (2012) el entorno familiar es la importancia que tiene la familia para el niño y la niña, especialmente para su desarrollo social, ya que el mismo representa el primer escenario de la socialización. El sistema familiar provee un espacio psicosocial en el que los niños y las niñas obtienen los elementos distintivos de la cultura y normas sociales que permiten su integración en la sociedad. Se concluye desde el punto de vista sociológico, que el entorno familiar es importante para el desarrollo de los niños y niñas en la sociedad, ya que sus comportamientos permiten su integración con la sociedad.

En este orden de ideas estos entornos, juegan un papel fundamental en el desarrollo, de las personas con discapacidad, siendo esto un problema de salud por parte del Estado ecuatoriano, en donde se limita de una o más capacidades físicas o biológicas, creando así una desigualdad en la sociedad. Se evidencia que, la mayoría de los sujetos no cuentan con el apoyo necesario por parte del Estado ecuatoriano, donde les ha tocado acomodarse al entorno sin lograr el respeto pleno de sus derechos humanos y sociales, establecidos en la Constitución y en los tratados internacionales. La condición de vida que llevan muchos de ellos, quienes en su mayoría son de escasos recursos económicos, no cuentan con los servicios básicos dentro de sus 
viviendas. Es importante que el Estado ecuatoriano tome cartas en el asunto para que puedan solucionarse este tipo de inconvenientes.

En el Estado ecuatoriano, existen normas que van en función de la preservación y defensa de los derechos de los niños, niñas y adolescentes con discapacidad, como un grupo de atención prioritaria, donde el entorno social y familiar juega un papel preponderante. El tema del entorno social y familiar de los niños con discapacidad es tema fundamental y que el mismo no es tomado en cuenta por parte de las autoridades del GAD Municipal del cantón Quevedo, a pesar de existir todo un marco jurídico enfocado a este aspecto. Por lo tanto, los derechos de los niños y niñas con discapacidad se encuentran garantizados tanto en la Constitución de la República del Ecuador, como en la normativa internacional, y se proponen campañas de concienciación a la ciudadanía, para dar a conocer los mismos y evitar así todo tipo de vulneración.

\section{FINANCIAMIENTO}

No monetario.

\section{AGRADECIMIENTO}

A la Universidad Regional Autónoma de los Andes, Quevedo, por motivar el desarrollo de la Investigación.

\section{REFERENCIAS CONSULTADAS}

Anabella Coria, B. J. (2012). El entorno familiar y su influencia en el plan de vida de los jóvenes. Intersiticios-Revista Sociológica de Pensamiento Crítico, 177-178.

Asamblea Nacional Constituyente de la República del Ecuador, (2008). Constitución de la República del Ecuador. [Constitution of the Republic of Ecuador]. Montecristi. Registro Oficial 449 de 20-oct-2008. Recuperado de https://n9.cl/sia

Asamblea Nacional del Ecuador (2012). Ley Orgánica de Discapacidades [Organic Law on Disabilities]. Publicada en Registro Oficial 796 del 25 de septiembre de 2012. Recuperado de: https://n9.cl/ratxu 
Ayala, M. (2020). Entorno social: características, elementos, influencia. [Social environment: characteristics, elements, influence] Recuperado de: https://n9.cl/vy0to

Comisión Nacional de los Derechos Humanos (2018) La Convención sobre los Derechos de las Personas con Discapacidad y su Protocolo Facultativo. [The Convention on the Rights of Persons with Disabilities and its Optional Protocol] Recuperado de: https://n9.cl/cnzw

Valencia, L. I. (2012). El contexto familiar: un factor determinante en el desarrollo social de los niños y las niñas. [The family context: a determining factor in the social development of children]. Recuperado de: Poiesis: https://n9.cl/o1vuf 